\title{
Enfoque diagnóstico de la tromboembolia pulmonar
}

\author{
Limberth Machado Villarroel, ${ }^{1}$ Diamanti Abraham Dimakis Ramírez ${ }^{2}$
}

\section{Resumen}

El embolismo pulmonar es una patología de presentación frecuente asociada a tasas de mortalidad elevadas; se caracteriza clínicamente por signos y síntomas altamente inespecíficos. Para su diagnóstico se cuenta con distintas modalidades, que comprenden escalas de predicción clínica (Wells y Ginebra modificadas), dímero-D, angiografía pulmonar por tomografía computarizada, angiografía por resonancia magnética, rayos $\mathrm{X}$, gammagrafía pulmonar de ventilación/perfusión, tomografía computarizada helicoidal, electrocardiograma, ecocardiograma y ecografía venosa. Anteriormente, otras pruebas - tales como la pletismografía de impedancia y la flebografía - resultaban de utilidad en el protocolo diagnóstico de la trombosis venosa profunda y, por ende, del embolismo pulmonar; empero, han sido desplazadas por técnicas que poseen mayor sensibilidad y especificidad. En fechas recientes se han desarrollado múltiples pruebas de laboratorio que cuantifican marcadores biológicos útiles en el diagnóstico del embolismo pulmonar, así como factores pronósticos; entre ellos están el péptido natriurético cerebral, proteína cardiaca de unión a los ácidos grasos, lipocalina asociada con la gelatinasa de los neutrófilos y cistatina C.

Palabras clave: Activador tisular del plasminógeno, angiografía pulmonar por tomografía computarizada, embolismo pulmonar, enfermedad tromboembólica venosa, heparinas de bajo peso molecular, heparina no fraccionada, filtros de vena cava inferior.

\begin{abstract}
Summary
Pulmonary embolism is a pathology of frequent presentation associated with high mortality rates; it is clinically characterized by highly unspecific signs and symptoms. Several modalities for its diagnosis exist: clinical prediction scores (revised Wells and Geneva scores), D-dimer, computed tomography pulmonary angiography, magnetic resonance angiography, X-rays, pulmonary ventilation/perfusion scan, helical computed tomography, electrocardiogram, echocardiogram and venous ultrasonography. Other tests, such as impedance plethysmography and phlebography were previously useful in the diagnostic protocol of deep vein thrombosis, and thus, pulmonary embolism; nevertheless, nowadays they have been displaced by tests with higher sensitivity and specificity. Several laboratory tests that measure biological markers useful in diagnosing pulmonary embolism have been developed; among them are brain natriuretic peptide, heart fatty acid binding protein, neutrophil gelatinaseassociated lipocalin and cystatin C.
\end{abstract}

Key words: Tissue plasminogen activator, computed tomography pulmonary angiography, pulmonary embolism, venous thromboembolic disease, low-molecular-weight heparins, unfractionated heparin, inferior vena cava filters.

\footnotetext{
${ }^{1}$ Especialista en Cardioneumología y Terapia Intensiva. Universidad Autónoma de Ciudad Juárez (UACJ). Hospital Ángeles Ciudad Juárez.

2 Médico interno de pregrado. Universidad Autónoma de Ciudad Juárez (UACJ).
}

Correspondencia:

Dr. Limberth Machado-Villarroel

Correo electrónico: limberthmv@hotmail.com

Aceptado: 19-10-2016.

Este artículo puede ser consultado en versión completa en http://www.medigraphic.com/actamedica 


\section{INTRODUCCIÓN}

La enfermedad tromboembólica venosa (ETV) comprende a la trombosis venosa profunda (TVP) y la embolia pulmonar (EP), padecimiento que se constituye como causa frecuente de muerte. Se trata de la tercera patología cardiovascular más frecuente después de la cardiopatía isquémica y la enfermedad cerebrovascular, con una tasa de incidencia anual de 100-200/100,000 habitantes; en personas mayores de 80 años de edad, la incidencia puede ser tan elevada como $1 / 100$ habitantes. ${ }^{1}$ Se estima que un poco más de 250,0000 personas fallecen anualmente en los Estados Unidos de América a causa de la ETV; 10-30\% de ellas mueren dentro del primer mes de efectuarse el diagnóstico. Se calcula que la tasa de mortalidad por EP no diagnosticada puede alcanzar un $30 \%$, mientras que sólo alrededor del $8 \%$ de los casos de EP diagnosticados y tratados oportunamente culminan en defunción. La ETV puede ser letal durante la fase aguda o generar patología crónica y discapacidad a largo plazo; no obstante, se trata de una entidad nosológica prevenible. La EP aguda (EPA) es la manifestación clínica más severa de la ETV. ${ }^{2}$

Resulta complicado establecer la epidemiología de la EP, pues en muchos casos es asintomática; sin embargo, la tasa de prevalencia estimada es del 1.5\%. En algunas circunstancias constituye un hallazgo incidental, mientras que en otras sólo se detecta postmortem, ya que 25\% de los casos de EP se presentan como muerte súbita. ${ }^{3}$ La EP constituye una causa importante de morbimortalidad y hospitalización a nivel mundial. Sin tratamiento, la EP tiene una tasa de mortalidad del $30 \%$, la cual puede reducirse a un $2-8 \%$ con un abordaje anticoagulante efectivo; de hecho, la EP se considera la causa evitable de muerte más común en pacientes hospitalizados. Se estima que una tercera parte de los individuos con EP presentarán recurrencia en los 10 años siguientes al momento del diagnóstico. ${ }^{4}$

\section{Factores de riesgo}

Existe una amplia gama de factores ambientales y genéticos que incrementan el riesgo de presentar ETV y, por ende, EP. ${ }^{5}$ Es bien sabido que ciertos trastornos genéticos predisponen a estados protrombóticos; los más frecuentes están representados por las mutaciones del factor $\mathrm{V}$ de Leiden (FVL) y del gen de protrombina $20210 .{ }^{6}$ Se calcula que la prevalencia de trombofilias hereditarias en la población general es del $0.2-0.4 \%$ por deficiencia de proteína C (PC), $0.2 \%$ por déficit de proteína $S$ (PS), $0.02 \%$ por déficit de antitrombina III (AT-III) y, por último, 4-5\% por mutaciones en el FVL. ${ }^{7}$ Se estima que un $11-29 \%$ de los sujetos con ETV presentan la mutación del FVL; asimismo, los portadores heterocigotos de dicha mutación poseen un riesgo 3-8 veces mayor de presentar ETV. La ETV se clasifica como poseedora de una causa aparente si existe un factor de riesgo temporal o reversible en un periodo de 1.5-6 semanas previas al diagnóstico, o sin causa aparente en caso de ausencia de él. Entre los factores de riesgo reversibles más destacables se encuentran cirugía, traumatismos, fracturas en miembros pélvicos, inmovilización prolongada, viajes largos en aeronaves, tabaquismo, obesidad, hipercolesterolemia, embarazo, uso de anticonceptivos orales y terapia de reemplazo hormonal durante la menopausia. ${ }^{6,7}$

Diversas situaciones clínicas actúan también como factores predisponentes en la EP; por ejemplo, Stojanovich y sus colaboradores observaron en un estudio clínico prospectivo que la tasa de prevalencia de EP en personas con síndrome antifosfolípido era del 12.3-13.2\%; dicha variación dependía de la subclase de anticuerpos antifosfolípidos expresados. ${ }^{8}$ Asimismo, la EP es una de las complicaciones más frecuentes y peligrosas en pacientes con lesión de médula espinal en fase aguda, presentándose en un $10-30 \%$ de los casos. ${ }^{9}$ La ETV con y sin EP asociada ocupa la segunda causa de mortalidad en individuos oncológicos, con una incidencia estimada del 10-15\% en dicho grupo de población. ${ }^{10}$ Mraovic y su grupo demostraron en un trabajo que el riesgo de EP era mayor en sujetos con síndrome metabólico postoperados de artroplastia de cadera o rodilla, e inclusive evidenciaron que la incidencia de EP incrementaba en relación con la cantidad de componentes del síndrome metabólico presentes en una persona determinada. ${ }^{11}$ Otras patologías y circunstancias clínicas en las cuales existe un riesgo elevado de EP son la hipertensión arterial sistémica, neumopatía obstructiva crónica, nefropatía crónica, diabetes mellitus y transfusión de hemoderivados. ${ }^{12}$ Cabe señalar que la EP puede llegar a presentarse incluso en ausencia total de factores de riesgo.

$$
\text { Fisiopatología }
$$

Al establecerse un episodio agudo de EP, se presentan múltiples anormalidades fisiopatológicas, las cuales se describen a continuación.

En primer lugar, debido a la oclusión de la permeabilidad de la arteria pulmonar, se altera la relación ventilación/ perfusión $(\mathrm{V} / \mathrm{Q})$ y, por lo tanto, se incrementa el espacio muerto fisiológico. Asimismo, se establece un cortocircuito derecha-izquierda (intrapulmonar o intracardiaco); lo anterior condiciona una respuesta refleja neurogénica que se traduce en hiperventilación alveolar. Por otro lado, se produce broncoconstricción en las vías aéreas distales al sitio de oclusión vascular, lo que conlleva un incremento de la resistencia al flujo aéreo. La EPA desencadena una disminución del factor surfactante, generalmente acompañada de hemorragia o edema, lo cual disminuye la 
distensibilidad pulmonar. Si el tromboembolismo ocluye más del 30-50\% del área de sección transversal del lecho arterial pulmonar, se produce un incremento de la presión arterial pulmonar sistólica (PAPS). ${ }^{13}$ Varias investigaciones han demostrado que la PAPS experimenta un incremento brusco dentro de los primeros 30 minutos posteriores al embolismo, alcanzando un valor máximo 1-2 horas después de dicho evento. Se estima que alrededor del $80 \%$ de los pacientes con TEP presentan hipertensión arterial pulmonar (HAP), mientras que un $0.5-4 \%$ desarrollarán hipertensión pulmonar tromboembólica crónica. ${ }^{14}$

La EP genera vasoconstricción a nivel local mediada por sustancias liberadas por plaquetas activadas tales como el tromboxano $\mathrm{A}_{2}\left(\mathrm{TxA}_{2}\right)$ y la serotonina, lo cual influye en el aumento inicial de la resistencia vascular pulmonar (RVP); asimismo, existe una disminución proporcional en la distensibilidad arterial. Múltiples estudios han demostrado que en las etapas iniciales de la EP existe una mayor síntesis de $\mathrm{TxA}_{2}$; asimismo, varios trabajos con modelos animales de TEP muestran una correlación entre la producción de dicha sustancia vasoconstrictora y un riesgo de mortalidad incrementado. ${ }^{15}$

Con el aumento súbito de la RVP se produce una dilatación del ventrículo derecho (VD), con la concomitante alteración de la contractilidad miocárdica e insuficiencia ventricular derecha (IVD). Se considera que la IVD debida a sobrecarga de presión es la principal causa de muerte en la EP severa. ${ }^{16}$ Cho y sus colegas concluyeron en un metaanálisis que la IVD demostrada mediante estudios ecocardiográficos acrecentaba la mortalidad por EPA a corto plazo hasta 2.29 veces. ${ }^{17}$ El alza en la presión y volumen del VD conlleva una subida en la tensión parietal y estiramiento miocítico, lo cual prolonga el tiempo de contracción del VD, mientras que una activación neurohumoral condiciona una estimulación cronotrópica e inotrópica. Dichos mecanismos compensatorios aumentan la PAPS, que mejora el flujo sanguíneo a través de la vasculatura pulmonar obstruida, estabilizando temporalmente la presión arterial sistémica. El alargamiento del periodo de contracción del VD conduce a un abombamiento del septo interventricular hacia el ventrículo izquierdo (VI) durante la fase inicial de la diástole; asimismo, se puede suscitar un bloqueo de la rama derecha del haz de His. Todo ello resulta en un defecto de llenado del VI, lo cual genera una reducción del gasto cardiaco que contribuye a la hipotensión sistémica e inestabilidad hemodinámica. Existe una elevación de los niveles plasmáticos de biomarcadores de daño miocárdico debido a isquemia del VD. ${ }^{18,19}$

\section{Cuadro clínico}

La EP puede ser una entidad patológica severa de difícil diagnóstico gracias a la inespecificidad de sus signos y síntomas, por lo que debe sospecharse en todo individuo con sintomatología cardiovascular. ${ }^{20}$ En una investigación realizada en el año 2011, Pollack y colaboradores fueron capaces de apreciar la frecuencia de los principales signos y síntomas de EP confirmado en sujetos que ingresaban al servicio de urgencias, los cuales se muestran a continuación por orden de frecuencia: 1) disnea $(50 \%), 2)$ dolor torácico de tipo pleurítico (39\%), 3) signos de TVP (24\%), 4) tos $(23 \%), 5)$ dolor torácico subesternal $(15 \%), 6)$ fiebre $(10 \%), 7)$ hemoptisis $\left(8 \%\right.$ ) y 8 ) síncope $(6 \%) .{ }^{21}$

Un hallazgo típico casi invariable de la EPA es la hipoxemia; sin embargo, hasta en un $40 \%$ de las personas la saturación de $\mathrm{O}_{2}$ en la gasometría arterial es normal; asimismo, hasta un $20 \%$ de los casos tienen un gradiente alvéolo-arterial de $\mathrm{O}_{2}$ normal. Otro resultado frecuente en la gasometría es la hipocapnia. ${ }^{22} \mathrm{Si}$ la presentación clínica sugiere $E P$, debe asumirse un enfoque diagnóstico objetivo. Resulta fundamental reconocer los factores de riesgo para ETV para determinar la probabilidad de EP; no obstante, hasta en un $30 \%$ de los casos no es posible identificar algún factor predisponente.

El abordaje diagnóstico actual para individuos en quienes se sospecha EP está basado en la valoración de las pruebas de probabilidad clínica; para esto, se emplean desde hace varios años diversas escalas de predicción clínica. Las escalas de Wells (Cuadro 1) y de Ginebra se han validado de forma extensa; estas utilizan un esquema de tres categorías (probabilidad clínica baja, moderada o alta para EP) y otro de dos categorías (probable o no probable

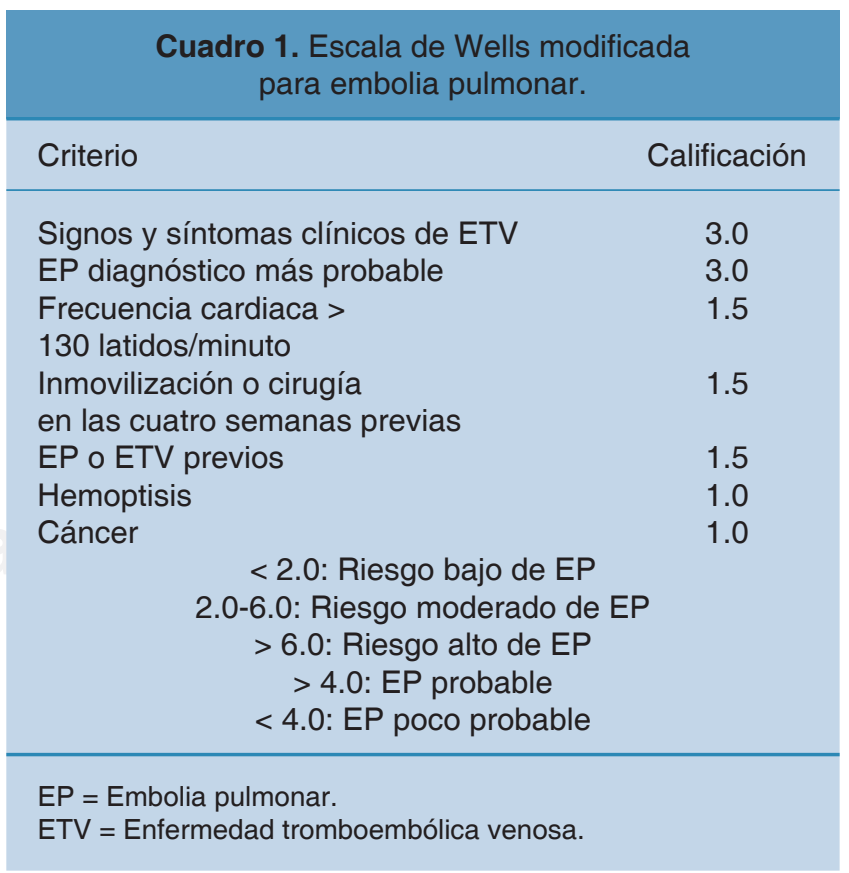


EP). Se estima que un $10 \%$ de los pacientes en la categoría de baja probabilidad clínica tendrán EP, 30\% en la categoría de probabilidad moderada y $65 \%$ en la categoría de probabilidad alta. ${ }^{23,24}$ Angriman y colaboradores evidenciaron en un estudio de individuos con EP subsegmentario que aquellos con una puntuación elevada en la escala de Wells al momento del diagnóstico de ETV presentaban un incremento de cuatro veces en la incidencia de muerte o episodio recidivante de ETV durante el seguimiento. ${ }^{25} \mathrm{Di}$ Marca y su grupo efectuaron un trabajo donde compararon las escalas de Wells y Ginebra como medidas predictivas de la probabilidad clínica de EP en sujetos ancianos hospitalizados y considerados de alto riesgo; encontraron que la escala de Wells tenía una mayor exactitud en dicho grupo de población. ${ }^{26}$

\section{DIAGNÓSTICO}

\section{Dímero-D}

Es la prueba diagnóstica más ampliamente utilizada en caso de sospecha de EP. El dímero-D es un marcador biológico cuya presencia es indicativa de formación y degradación de fibrina; se detecta en plasma tras la fibrinólisis de un coágulo. Posee una elevada sensibilidad (98-100\%) y baja especificidad (35-39\%), mientras que el valor predictivo negativo (VPN) es del 95\%. Por lo anterior, la prueba de dímero-D únicamente tiene utilidad para descartar EP en sujetos con probabilidad clínica baja o intermedia con una puntuación $\leq 4$ en las escalas de predicción clínica y niveles séricos de dímero-D $\leq 500 \mu \mathrm{g} / \mathrm{L}$; dicha combinación se presenta en $30-40 \%$ de las personas con sospecha de EP. ${ }^{27,28}$ La reducida especificidad del dímero-D para el diagnóstico de ETV, especialmente en pacientes ancianos, se debe a que se observan concentraciones plasmáticas elevadas de dímero-D en individuos con procesos inflamatorios extensos, heridas en proceso de cicatrización, cáncer, hepatopatía, traumatismos y cirugías, etcétera. ${ }^{29}$

Se hace valorable el dímero-D principalmente cuando se emplea en conjunto con escalas de predicción clínica que dictaminan una probabilidad clínica baja o intermedia. ${ }^{30,31}$ Se ha demostrado que menos del $5 \%$ de los sujetos mayores de 80 años de edad tienen un valor negativo en la prueba del dímero-D, por lo que la relación costo-beneficio de dicho ensayo diagnóstico en la población geriátrica es desfavorable. Sin embargo, un dato a destacar es que por una parte, un dímero-D dentro de parámetros normales descarta el diagnóstico de TVP y EP en aquellas personas con una probabilidad clínica baja de ETV. ${ }^{32,33}$

El dímero-D no constituye una simple molécula homogénea; por tanto, la mayor parte de los problemas de cuantificación del mismo emergen de su heterogeneidad bioquímica. A manera de resumen, el dímero-D es el producto de la degradación de entrecruzamientos de fibrina y, por ende, refleja la activación de procesos como la hemostasia y fibrinólisis. ${ }^{34}$ La degradación de la molécula de fibrina mediada por plasmina, en la cual dos partículas D adyacentes se han entrecruzado por activación del factor XIII (FXIII) de la coagulación, genera una variedad de fragmentos que contienen el dominio-D dimérico (Figura 1). ${ }^{35}$ La presencia del dímero-D puede ser detectada utilizando anticuerpos monoclonales cuyo blanco específico consiste en un epítopo presente en el fragmento de FXIII activado entrecruzado con el dominio-D de la fibrina, más no así en los productos de la degradación del fibrinógeno o en los productos de la degradación de la fibrina no entrecruzados. El resultado final de la degradación de la fibrina es, por tanto, una clase heterogénea de péptidos caracterizados por la presencia de múltiples dominios-D entrecruzados. ${ }^{36,37}$

\section{Angiografía pulmonar por tomografía computarizada}

La angiografía pulmonar por tomografía computarizada (APTC) constituye la prueba de imagen diagnóstica más utilizada para EP en pacientes hemodinámicamente estables. ${ }^{38,39}$ Resulta evidentemente superior a diferentes métodos de imagen y es considerada un excelente método diagnóstico al permitir apreciar las dimensiones de las cámaras cardiacas y la visualización directa de la circulación arterial pulmonar posteriormente a la inyección intravenosa (IV) de un medio de contraste yodado; en muchos casos, es capaz de detectar hallazgos incidentales en la mayoría de los individuos, los cuales ocasionalmente permiten explicar su sintomatología. 40,41 La APTC genera imágenes de alta resolución que son capaces de visualizar elementos de sexto orden de la vasculatura pulmonar. Un marcador importante de IVD es el índice o relación $\mathrm{VD} / \mathrm{VI}$; se ha establecido que aquellos sujetos en quienes se realiza la APTC y se determina una relación ventrículo derecho/ventrículo izquierdo $\geq 0.9$ presentan un riesgo mayor de mortalidad dentro del primer mes de la EP, por lo que proporciona información valiosa en términos pronósticos. ${ }^{42}$

Entre sus ventajas están el ser un método no invasivo y de rápida realización que posee una sensibilidad y especificidad elevadas (96-100\% y 97-98\%, respectivamente), sobre todo en los modernos dispositivos de tecnología multicorte. Sin embargo, se deben mencionar desventajas de esta modalidad diagnóstica, ya que la utilización de radiación ionizante y el riesgo de nefropatía inducida por el medio de contraste es de $4 \%{ }^{43,44}$ Se ha reportado en diversas investigaciones que la APTC incrementa el riesgo de padecer cáncer de mama hasta en un 14\% después de la administración de una dosis de 10 mGy. ${ }^{45}$ 


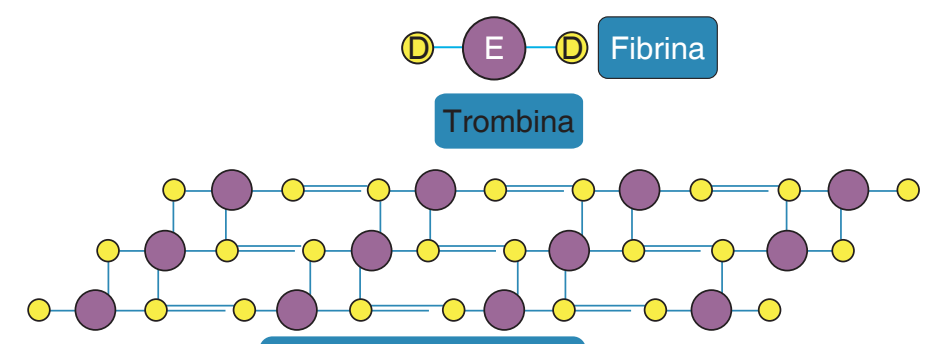

Malla de fibrina

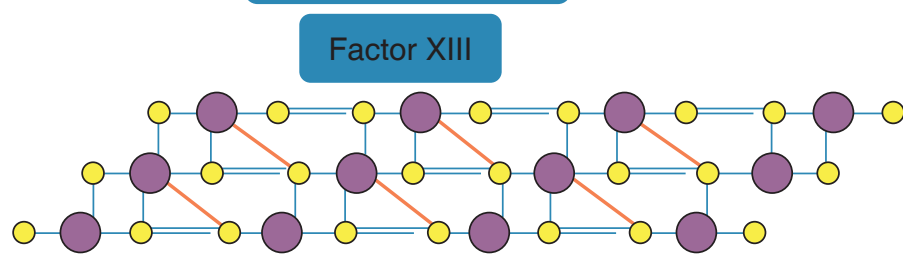

Malla de fibrina entrecruzada

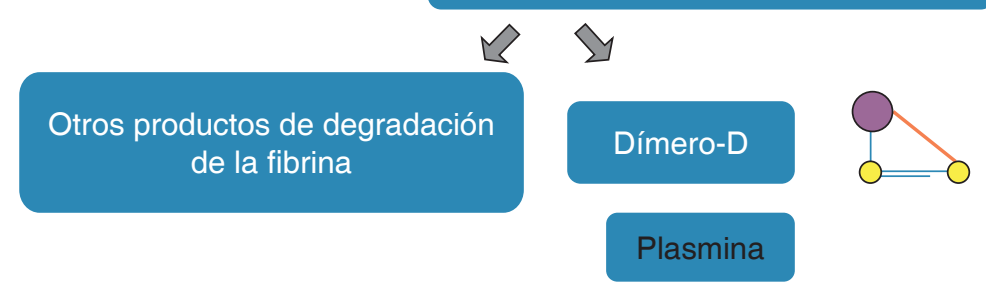

Figura 1.

Formación del dímero-D y otros productos de degradación de la fibrina. Traducido y adaptado con permiso de Eur Heart $J$ Cardiovasc Pharmacother [Soomro AY, Guerchicoff A, Nichols DJ, Suleman $J$, Dangas GD. The current role and future prospects of D-dimer biomarker. Eur Heart $J$ Cardiovasc Pharmacother. 2(3): 175-184], derechos reservados (2016).
La APTC deberá ser utilizada en combinación con una escala de predicción clínica. Los criterios diagnósticos aceptados para EP por APTC son: 1) ausencia de opacificación del lumen vascular a expensas de un defecto de llenado central, 2) un defecto de llenado parcial rodeado por material de contraste en un corte transversal, 3) la presencia de material de contraste entre el defecto de llenado central y la pared arterial en un corte longitudinal o 4) un defecto intraluminal periférico que forma un ángulo agudo con la arteria. ${ }^{46,47}$ Se ha demostrado en varios estudios que una APTC con resultado negativo descarta de manera segura el diagnóstico de EP, puesto que existe un riesgo muy bajo de ETV a los tres meses en personas sin tratamiento después de una APTC negativa (1.2\%, [intervalo de confianza de 95\%, 0.8-1.8]). ${ }^{48}$

\section{Angiografía por resonancia magnética (ARM)}

La imagen por resonancia magnética (IRM) ha sido recientemente evaluada por algunos investigadores como una técnica alternativa para el diagnóstico de la EP; empero, aún no se recomienda su empleo aislado como prueba diagnóstica; por ello, se combina con otras técnicas no invasivas como la angiografía pulmonar. ${ }^{49}$ La ARM posee una elevada sensibilidad (77-100\%) para el diagnóstico de la EP proximal; sin embargo, se ha reportado una baja sensibilidad en el diagnóstico de la misma ante la segmentación progresiva y extensión distal de la arteria pulmonar (84\% en las arterias segmentarias y $40 \%$ en las subsegmentarias), así como una tasa elevada de estudios técnicamente inadecuados. Por otro lado, hay reportes donde la IRM ha demostrado tener una alta especificidad (97.4-100\%) en el diagnóstico de la EP; sin embargo, no son resultados contundentes. ${ }^{50}$

Es bien sabido que la IRM presenta gran susceptibilidad a los artefactos, lo cual podría limitar su uso; a pesar de las implementaciones técnicas, la tasa de resultados inconclusos llega a alcanzar valores de hasta un 30\%. Otra de las limitaciones en el uso de la IRM es el riesgo inherente de fibrosis sistémica nefrogénica inducida por medios de contraste a base de gadolinio, tales como la gadodiamida y el gadopentetato de dimeglumina, en pacientes con insuficiencia renal. ${ }^{51}$ Otras desventajas de esta modalidad diagnóstica son la duración prolongada del proceso de examinación y la baja disponibilidad del equipo en cuestión, sobre todo en el escenario de urgencias hospitalarias. Actualmente, la ARM no está recomendada como opción principal y para su realización se sugiere en centros hospitalarios que cuenten con personal altamente especializado y capacitado, así como 
en aquellos individuos con alguna contraindicación para las pruebas estándar. ${ }^{52}$

\section{Gammagrafía pulmonar de ventilación/perfusión}

La gammagrafía pulmonar estudia dos funciones esenciales: perfusión pulmonar y ventilación. Dicha técnica involucra comúnmente la demostración de perfusión pulmonar empleando un bloqueo capilar limitado a través de partículas radioactivas inyectadas (microesferas de albúmina humana o microagregados de albúmina marcados con tecnecio-99m [99mTc-MAH y ${ }^{99 m}$ Tc-MAA, respectivamente]), así como la evaluación del intercambio gaseoso utilizando gases o aerosoles inertes inhalados $\left({ }^{81 \mathrm{~m}} \mathrm{Kr}\right.$ y ácido dietilentriaminopentaacético marcado con tecnecio-99m [ ${ }^{99 m}$ Tc-ADTP], entre otros). ${ }^{53}$ Se trata de una prueba esencialmente cualitativa que muestra una ventaja sobre la mayoría de las pruebas cuantitativas de la función pulmonar global al ser capaz de distinguir entre neumopatía difusa y regional, con la opción de una semicuantificación locorregional. ${ }^{54}$

La gammagrafía de ventilación/perfusión resulta una herramienta diagnóstica de utilidad para la detección y seguimiento clínico de la EP. La visualización de la vía aérea regional y la integridad vascular constituyen el fundamento del diagnóstico gammagráfico de dicha entidad, siendo a la vez su principal indicación. La gammagrafía pulmonar tiene ciertas ventajas en comparación con la tomografía computarizada helicoidal $(\mathrm{TCH})$, sobre todo en lo concerniente a la detección de émbolos pulmonares más periféricos y de menores dimensiones, así como la necesidad de una menor dosis de radiación. Adicionalmente, resulta factible descubrir de forma incidental hallazgos indicativos de otras neumopatías, tales como enfermedad pulmonar obstructiva crónica (EPOC), neumonía e insuficiencia cardiaca izquierda, entre otras. ${ }^{55}$

Las anomalías ventilatorias comúnmente ocasionan una redistribución de la perfusión pulmonar. La hipoventilación condiciona hipoxia regional y una redistribución refleja de la perfusión distante a las regiones hipoventiladas. ${ }^{56} \mathrm{Se}$ recomienda contar con una radiografía de tórax en dos proyecciones distintas al momento de la gammagrafía para definir o excluir la presencia de infiltrados, derrame pleural, neumotórax o lesiones ocupantes de espacio al interior del tórax. Siempre que sea posible, se deberá adicionar una prueba simple de función pulmonar, como la espirometría con parámetros ventilatorios forzados. ${ }^{5}$

Tomografía computarizada helicoidal (TCH)

Con esta modalidad se obtiene una imagen volumétrica bidimensional del sujeto mediante un detector rotacional. ${ }^{57}$
Los émbolos pulmonares se aprecian como defectos de llenado en la circulación pulmonar, pueden tener una localización central, mural o excéntrica y ocluir parcial o totalmente una arteria pulmonar. De forma adicional, la $\mathrm{TCH}$ proporciona valor pronóstico al evaluar las dimensiones del VD y, en ciertos casos, establecer diagnósticos alternativos. ${ }^{58}$ Esta prueba de imagen tiene reportada una sensibilidad para el diagnóstico de EP de 53-100\%, generalmente baja en caso de obstrucción de arterias subsegmentarias periféricas; asimismo las especificidades van del 81 al $100 \%{ }^{59}$

La TCH con medio de contraste se ha convertido en la modalidad de imagen preferida en el caso de sospecha de EP en muchos hospitales, pues su resultado es inequívoco, se demuestra o descarta la presencia de un trombo. Tiene la desventaja de no encontrarse disponible en todos los centros hospitalarios. Cabe destacar su contraindicación en personas con insuficiencia renal $y / 0$ alergia al medio de contraste. ${ }^{60}$

La TCH no debe utilizarse como prueba única para descartar la presencia de EP, puesto que no se ha determinado la seguridad clínica de retardar la terapia anticoagulante tras un estudio con resultados normales. La TCH resulta útil en combinación con otras pruebas diagnósticas, particularmente después de una primera ronda diagnóstica que haya incluido escalas de probabilidad clínica y determinación de dímero-D. ${ }^{61}$

\section{Rayos X}

Se trata de una modalidad diagnóstica con baja sensibilidad; puede llegar a ser normal inclusive en pacientes con una clínica altamente sugestiva de EP. La radiografía torácica cobra importancia para corroborar o excluir el diagnóstico de otras entidades patológicas con un cuadro clínico similar a la EP. En un trabajo de Abbas y colaboradores se demostró que el signo radiológico de Westermark (oligohemia focal con hipertransparencia pulmonar) estaba presente en $8-14 \%$ de los casos confirmados de EP. Otros hallazgos radiológicos típicos de la EP son el signo de Fleischner (dilatación de la arteria pulmonar en el sitio de enclavamiento del émbolo) y la joroba de Hampton (condensación parenquimatosa focal segmentaria cuneiforme con base pleural y vértice central, correspondiente a la zona de infarto pulmonar). Asimismo, el signo de Palla (rama descendente de la arteria pulmonar derecha prominente) representa un posible hallazgo imagenológico. Por último, existen datos radiográficos inespecíficos que, no obstante, pueden evidenciarse en la EP, tales como la elevación del hemidiafragma y reducción del volumen del pulmón afectado, imágenes de derrame pleural y atelectasias laminares. ${ }^{62-64}$ 


\section{Electrocardiograma (ECG)}

El ECG es generalmente anormal en caso de EP; sin embargo, sus hallazgos no son lo suficientemente sensibles o específicos. Por ello, resulta particularmente útil para descartar otras patologías que también ponen en riesgo la vida, como el infarto agudo al miocardio (IAM). Es posible identificar los siguientes hallazgos electrocardiográficos: taquicardia sinusal, bloqueo completo/incompleto de la rama derecha del haz de His, ondas $\mathrm{T}$ invertidas en derivaciones precordiales derechas (V1, V2, V3, V4, DII, DIII y aVF), dextrorrotación del eje cardiaco, onda $R$ dominante en $\mathrm{V} 1$, hipertrofia atrial derecha (onda P pulmonale), onda $\mathrm{S}$ persistente en $\mathrm{V} 6$, taquiarritmias atriales y elevación/descenso del segmento ST. Cabe destacar que Zhan y colaboradores objetivaron en una investigación la presencia del patrón electrocardiográfico $\mathrm{S}_{\mathrm{I}}-\mathrm{Q}_{\mathrm{III}}{ }^{-\mathrm{T}_{\text {III }}}$ (complejo de McGinn-White) en 15-25\% de los individuos con EP confirmada. ${ }^{65-67}$

\section{Ecocardiograma}

El ecocardiograma es un método diagnóstico capaz de definir el grado de repercusión funcional del VD secundaria a TEP; asimismo, posee implicaciones terapéuticas y pronósticas, pues permite determinar las dimensiones de las cavidades cardiacas derechas y calcular la PAPS. ${ }^{68}$ Se ha reportado un VPN para el ecocardiograma del 40-50\%; por ello, un resultado negativo no excluye el diagnóstico de EP. Por otro lado, los signos de sobrecarga o IVD también pueden observarse aun en la ausencia de EP y estar condicionados por alguna cardiopatía/neumopatía subyacente. ${ }^{69}$

Los siguientes son posibles hallazgos ecocardiográficos en la EP: ${ }^{70}$

- Dilatación ventricular derecha en alrededor del 25\% de los casos.

- Contractilidad disminuida de la pared libre del VD en comparación con su ápex (signo de McConnell).

- Trombos móviles en corazón derecho (detectados sobre todo mediante ecocardiografía transtorácica o transesofágica).

La exploración ecocardiográfica es un requisito de la evaluación diagnóstica en sujetos hemodinámicamente estables, normotensos, con sospecha de bajo riesgo para EP. En caso de sospecha de alto riesgo para EP, la ausencia de signos ecocardiográficos de sobrecarga ventricular derecha o insuficiencia prácticamente excluye a la EP como la causa de la inestabilidad hemodinámica. ${ }^{71}$
Ecografía venosa (EV)

En la mayoría de los casos, la EP se origina a partir de una TVP en un miembro pélvico. Actualmente, la EV ha reemplazado a la venografía como prueba diagnóstica de TVP, con una sensibilidad mayor al $90 \%$ y especificidad del $95 \%$ para TVP sintomática. La EV demuestra la presencia de TVP en $30-50 \%$ de las personas con EP; asimismo, encontrar TVP proximal en pacientes con sospecha de EP constituye una indicación para iniciar la terapia anticoagulante sin necesidad de realizar pruebas adicionales. ${ }^{72} \mathrm{Sin}$ embargo, en múltiples estudios clínicos retrospectivos y prospectivos se ha encontrado que solamente $0-22 \%$ de los individuos con diagnóstico de EP presentan de forma simultánea TVP en las extremidades inferiores. ${ }^{73}$

En caso de sospecha de EP, la EV se puede limitar a una simple exploración en cuatro puntos (regiones inguinales y fosas poplíteas). El único criterio diagnóstico validado para TVP es la compresibilidad incompleta de la vena, lo cual señala la presencia de un coágulo, mientras que las mediciones de flujo se consideran poco confiables. El rendimiento diagnóstico de la EV en EP sospechada puede incrementarse mediante la realización de una ultrasonografía completa que incluya también las venas distales. ${ }^{74}$

\section{Pletismografía de impedancia (PI)}

La PI es una técnica no invasiva establecida para detectar trombosis proximal (venas poplíteas, femorales e iliacas) en sujetos con un primer episodio de trombosis venosa clínicamente sospechada. Tiene como finalidad cuantificar los cambios en el volumen sanguíneo del miembro pélvico en términos de modificaciones en la resistencia eléctrica; es decir, la impedancia. Dicho instrumento provee una corriente eléctrica débil y constante que pasa a través de la pantorrilla y es detectada mediante electrodos situados en la región crural. ${ }^{75}$ Las variaciones en el volumen sanguíneo son producidas por la insuflación de un manguito crural a una presión que excede la presión venosa pero es menor a la presión arterial diastólica. Al dejar de ejercer presión mediante dicho manguito, se debe presentar un flujo venoso rápido en los miembros pélvicos normales; sin embargo, en presencia de trombosis venosa, el vaciamiento venoso es lento. Con el advenimiento de la EVC, la PI ha caído en desuso. ${ }^{76}$

\section{Flebografía}

La flebografía con contraste representa el método diagnóstico tradicional de TVP. Más recientemente, se ha implementado la flebografía por medicina nuclear, la cual emplea fibrinógeno marcado. A semejanza de la Pl, la fle- 
bografía ha sido desplazada gradualmente por los métodos no invasivos. ${ }^{77}$

\section{Pruebas de laboratorio y marcadores biológicos}

La EP está caracterizada por anormalidades en el intercambio gaseoso. Es bien sabido que el establecimiento de un cortocircuito intrapulmonar es el principal factor generador de hipoxemia y disminución de la $\mathrm{PaO}_{2}$ en la EP. Diversos trabajos han demostrado que la leucocitosis contribuye como un factor diagnóstico y pronóstico en personas con EP, ya que un contexto clínico y un conteo de glóbulos blancos $\geq$ a $11,000 / \mathrm{mm}^{3}$ están relacionados con un incremento en la mortalidad durante los primeros 30 días de ingreso hospitalario. ${ }^{78}$

Las dimensiones del émbolo, el grado de obstrucción del lumen vascular, el tiempo de embolización y la patología cardiopulmonar subyacente son distintos factores que eventualmente determinan el grado de afección del intercambio gaseoso. Por otro lado, durante la EPA se presenta una respuesta compensatoria de hiperventilación, la cual eventualmente ocasiona hipocapnia y disminución de la $\mathrm{PaCO}_{2}$, con la alcalosis respiratoria concomitante. ${ }^{79}$ Conforme progresa la EP, es posible detectar incremento en los niveles séricos de la enzima lactato deshidrogenasa; esto sugiere daño celular y/o necrosis. ${ }^{80}$

La proteína cardiaca de unión a los ácidos grasos (PCUAG) es una proteína citosólica de bajo peso molecular que se detecta en el torrente sanguíneo tras un IAM; en el contexto de una EPA, la tensión incrementada en el VD ocasiona daño miocárdico y la liberación subsecuente de PCUAG. Investigaciones previas han demostrado que la PCUAG posee una sensibilidad y VPN elevados, lo cual permite estratificar de forma temprana a la EPA con mayor riesgo de eventos adversos y/o mortalidad. ${ }^{81}$ Otros marcadores de daño miocárdico como la troponina $\mathrm{T}$, péptido natriurético cerebral (PNC) y el fragmento $\mathrm{N}$-terminal de su prohormona (NT-proPNC) se consideran actualmente biomarcadores vigentes y muy útiles en pacientes con EP. ${ }^{82}$ El PNC y NT-pro PNC son indicadores altamente sensibles de IVD, por lo que pueden emplearse con fines de exclusión de mortalidad elevada o complicaciones en caso de EPA. ${ }^{83-85}$

La lipocalina asociada con la gelatinasa de los neutrófilos (LAGN) es una sustancia implicada en la formación de nefronas en el riñón embrionario; su síntesis se ve incrementada de forma masiva ante una lesión renal aguda (LRA). Kostrubiec y colaboradores demostraron en un estudio que hasta un 30\% de los individuos con EPA presentan datos de LRA, lo cual se encuentra asociado con un incremento de los niveles séricos de LAGN. La cistatina $C$ es un marcador endógeno de disfunción renal, se detecta en el plasma antes del incremento de la creatinina. Asimismo, la elevación de los niveles de cistatina $\mathrm{C}$ y una reducción en la tasa de filtrado glomerular (TFG) están asociadas con un peor pronóstico de la EPA en los primeros 30 días. ${ }^{86}$

\section{CONCLUSIONES}

La EP constituye la presentación clínica más severa de la ETV. La mayoría de las veces, la EP es consecuencia de la TVP; por ello, la información existente en la actualidad respecto a su epidemiología, factores de riesgo e historia natural de la enfermedad se encuentra determinada por trabajos que analizan a la ETV de forma general. El cuadro clínico de la EP puede ser muy inespecífico; por tanto, los sujetos con sospecha de dicha patología deben ser sometidos a múltiples estudios de laboratorio y gabinete capaces de confirmarla o, en su defecto, plantear diagnósticos diferenciales. Existen diversas herramientas diagnósticas con utilidad en la EP, como escalas de predicción clínica (Wells y Ginebra modificadas), dímero-D, gasometría arterial, citometría hemática completa, determinación sérica de troponina y/o PNC, angiografía, flebografía, TCH, IRM, radiografía torácica, gammagrafía pulmonar de ventilación/ perfusión, electrocardiografía, ecocardiografía y ecografía venosa, entre otras.

\section{REFERENCIAS}

1. Li F, Wang X, Huang W, Ren W, Cheng J, Zhang M et al. Risk factors associated with the occurrence of silent pulmonary embolism in patients with deep venous thrombosis of the lower limb. Phlebology. 2014; 29 (7): 442-446.

2. Guijarro R, Trujillo-Santos J, Bernal-Lopez MR, de Miguel-Díez J, Villalobos A, Salazar C et al. Trend and seasonality in hospitalizations for pulmonary embolism: a time-series analysis. J Thromb Haemost. 2015; 13: 23-30.

3. Bach AG, Taute BM, Baasai N, Wienke A, Meyer HJ, Schramm D et al. 30-day mortality in acute pulmonary embolism: prognostic value of clinical scores and anamnestic features. PLoS One. 2016; 11 (2): e0148728.

4. Goncharova EA, Gladwin MT, Kawut SM. Update in pulmonary vascular diseases 2014. Am J Respir Crit Care Med. 2015; 192 (5): 544-550.

5. Donadini MP, Dentali F, Squizzato A, Guasti L, Ageno W. Unsuspected pulmonary embolism in cancer patients: a narrative review with pooled data. Intern Emerg Med. 2014; 9 (4): 375-384.

6. Ali N, Ayyub M, Khan SA. High prevalence of protein C, protein S, antithrombin deficiency, and factor $\mathrm{V}$ Leiden mutation as a cause of hereditary thrombophilia in patients of venous thromboembolism and cerebrovascular accident. Pak J Med Sci. 2014; 30 (6): 1323-1326.

7. Jun ZJ, Ping T, Lei Y, Li L, Ming SY, Jing W. Prevalence of factor V Leiden and prothrombin G20210A mutations in Chinese patients with deep venous thrombosis and pulmonary embolism. Clin Lab Haematol. 2006; 28 (2): 111-116.

8. Stojanovich L, Kontic M, Djokovic A, Ilijevski N, Stanisavljevic N, Marisavljevic D. Pulmonary events in antiphospholipid syndrome: influence of antiphospholipid antibody type and levels. Scand J Rheumatol. 2012; 41 (3): 223-226. 
9. Aito S, Pieri A, D'Andrea M, Marcelli F, Cominelli E. Primary prevention of deep venous thrombosis and pulmonary embolism in acute spinal cord injured patients. Spinal Cord. 2002; 40 (6): 300-303.

10. Sawant SP, Banumathy S, Daddi A, Dhir AA. Pulmonary embolism in cancer patients. Indian J Cancer. 2012; 49 (1): 119-124.

11. Mraovic B, Hipszer BR, Epstein RH, Parvizi J, Pequignot EC, Chervoneva I et al. Metabolic syndrome increases risk for pulmonary embolism after hip and knee arthroplasty. Croat Med J. 2013; 54 (4): 355-361.

12. Zahir U, Sterling RS, Pellegrini VD, Forte ML. Inpatient pulmonary embolism after elective primary total hip and knee arthroplasty in the United States. J Bone Joint Surg Am. 2013; 95 (22): e175.

13. Jing ZC. China's guidelines on the diagnosis and treatment of pulmonary hypertension. Chin J Frontiers Med Sci. 2011; 2: 62-81.

14. Liu YY, Li XC, Duan Z, Yuan YD. Correlation between the embolism area and pulmonary arterial systolic pressure as an indicator of pulmonary arterial hypertension in patients with acute pulmonary thromboembolism. Eur Rev Med Pharmacol Sci. 2014; 18 (17): 25512555.

15. Smulders YM. Pathophysiology and treatment of haemodynamic instability in acute pulmonary embolism: the pivotal role of pulmonary vasoconstriction. Cardiovasc Res. 2000; 48 (1): 23-33.

16. Deng X, Li Y, Zhou L, Liu C, Liu M, Ding N et al. Gender differences in the symptoms, signs, disease history, lesion position and pathophysiology in patients with pulmonary embolism. PLoS One. 2015; 10 (7): e0133993.

17. Cho JH, Kutti Sridharan G, Kim SH, Kaw R, Abburi T, Irfan A et al. Right ventricular dysfunction as an echocardiographic prognostic factor in hemodynamically stable patients with acute pulmonary embolism: a meta-analysis. BMC Cardiovasc Disor. 2014; 14: 64.

18. Montagnana M, Cervellin G, Franchini M, Lippi G. Pathophysiology, clinics and diagnostics of non-thrombotic pulmonary embolism. J Thromb Thrombolysis. 2011; 31 (4): 436-444.

19. Banks DA, Pretorius GV, Kerr KM, Manecke GR. Pulmonary endarterectomy: Part I. Pathophysiology, clinical manifestations, and diagnostic evaluation of chronic thromboembolic pulmonary hypertension. Semin Cardiothorac Vasc Anesth. 2014; 18 (4): 319-330.

20. Sinha N, Padegal V, Satyanarayana S, Santosh HK. Pulmonary cement embolization after vertebroplasty, an uncommon presentation of pulmonary embolism: a case report and literature review. Lung India. 2015; 32 (6): 602-605.

21. Pollack CV, Schreiber D, Goldhaber SZ, Slattery D, Fanikos J, O'Neil BJ et al. Clinical characteristics, management, and outcomes of patients diagnosed with acute pulmonary embolism in the emergency department: initial report of EMPEROR (Multicenter Emergency Medicine Pulmonary Embolism in the Real World Registry). J Am Coll Cardiol. 2011; 57 (6): 700-706.

22. Abdalla A, Kelly F. "STEMI-like" acute pulmonary embolism, an unusual presentation. BMJ Case Rep. 2014; 2014. pii: bcr2014206517. doi: 10.1136/bcr-2014-206517.

23. Modi S, Deisler R, Gozel K, Reicks P, Irwin E, Brunsvold M et al. Wells criteria for DVT is a reliable clinical tool to assess the risk of deep venous thrombosis in trauma patient. World J Emerg Surg. 2016; 11: 24.

24. Bertoletti L, Le Gal G, Aujesky D, Roy PM, Sánchez O, Verschuren F et al. Prognostic value of the Geneva prediction rule in patients in whom pulmonary embolism is ruled out. J Intern Med. 2011; 269 (4): 433-440.

25. Angriman F, Ferreyro BL, Posadas-Martínez ML, Giunta D, Vázquez FJ, Vollmer WM. Wells score and poor outcomes among adult patients with subsegmental pulmonary embolism: a cohort study. Clin Appl Thromb Hemost. 2015; 21 (6): 539-545.

26. Di Marca S, Cilia C, Campagna A, D’Arrigo G, Abd ElHafeez S, Tripepi $G$ et al. Comparison of Wells and revised Geneva rule to assess pretest probability of pulmonary embolism in high-risk hospitalized elderly adults. J Am Geriatr Soc. 2015; 63 (6): 10911097.

27. Nemenqani D, Fayek MH, Ahmad SA, Elnashar H, Asaad H. Validation of new D-dimer cutoff values to increase its diagnostic utility as biochemical marker in acute venous thromboembolic disease. Egypt J Hosp Med. 2015; 61: 363-370.

28. Lippi G, Cervellin G, Casagranda I, Morelli B, Testa S, Tripodi A. D-dimer testing for suspected venous thromboembolism in the emergency department. Consensus document of AcEMC, CISMEL, SIBioC, and SIMeL. Clin Chem Lab Med. 2014; 52 (5): 621-628.

29. Michiels J], Maasland H, Moossdorff W, Lao M, Gadiseur A, Schroyens W. Safe exclusion of deep vein thrombosis by a rapid sensitive ELISA D-dimer and compression ultrasonography in 1,330 outpatients with suspected DVT. Angiology. 2016; 67 (8): 781-787.

30. Kearon C, Spencer FA, O'Keeffe D, Parpia S, Schulman S, Baglin T et al. D-dimer testing to select patients with a first unprovoked venous thromboembolism who can stop anticoagulant therapy: a cohort study. Ann Intern Med. 2015; 162: 27-34.

31. Stein PD, Matta F, Sabra MJ, Tana C, Gough A, Chabala S et al. Specificity of quantitative latex agglutination assay for D-dimer in exclusion of pulmonary embolism in the emergency department. Clin Appl Thromb Hemost. 2014; 20 (8): 807-812.

32. Halaby R, Popma CJ, Cohen A, Chi G, Zacarkim MR, Romero G et al. D-dimer elevation and adverse outcomes. J Thromb Thrombolysis. 2015; 39: 55-59.

33. Shah K, Quaas J, Rolston D, Bansal S, Banja T, Newman D et al. Magnitude of D-dimer matters for diagnosing pulmonary embolus. Am J Emerg Med. 2013; 31: 942-945.

34. Crop MJ, Siemes C, Berendes P, van der Straaten F, Willemsen S, Levin $\mathrm{MD}$. Influence of $\mathrm{C}$-reactive protein levels and age on the value of D-dimer in diagnosing pulmonary embolism. Eur J Haematol. 2013; 92 (2): 147-155.

35. Le Gal G, Righini M, Wells PS. D-dimer for pulmonary embolism. JAMA. 2015; 313 (16): 1668-1669.

36. Cohen AT, Spiro TE, Spyropoulos AC, DeSanctis YH, Homering M, Büller HR et al. D-dimer as a predictor of venous thromboembolism in acutely ill, hospitalized patients: a subanalysis of the randomized controlled MAGELLAN trial. J Thromb Haemost. 2014; 12 (4): 479-487.

37. Cini M, Legnani C, Frascaro M, Sartori M, Cosmi B, Palareti G. D-dimer use for deep venous thrombosis exclusion in elderly patients: a comparative analysis of three different approaches to establish cutoff values for an assay with results expressed in D-dimer units. Int J Lab Hematol. 2014; 36 (5): 541-547.

38. Le Gal G, Righini M, Wells PS. Computed tomographic pulmonary angiography for pulmonary embolism. J Am Med Assoc. 2015; 314 (1): 74-75.

39. Javadrashid R, Mozayan M, Tarzamni MK, Ghaffari MR, Fouladi DF. Spiral computed tomographic pulmonary angiography in patients with acute pulmonary emboli and no pre-existing comorbidity: a prospective prognostic panel study. Eur Radiol. 2015; 25: 147-154.

40. Perelas A, Dimou A, Saenz A, Rhee JH, Teerapuncharoen K, Rowden A et al. Incidental findings on computed tomography angiography in patients evaluated for pulmonary embolism. Ann Am Thorac Soc. 2015; 12 (5): 689-695.

41. Allen C, Seinge R, Maxwell R, Thind D. CT pulmonary angiography and pulmonary embolism following 5,809 primary joint arthroplasties. N Z Med J. 2015; 128 (1413): 41-49.

42. Morales-Borrero WJ, Maldonado-Vargas JA. Diagnostic yield of pulmonary CT angiography in the evaluation of pulmonary embolisms treated at the Puerto Rico Medical Center from 2008 to 2012. P R Health Sci J. 2016; 35 (1): 16-19. 
43. Minshall CT, Doben AR, Leon SM, Fakhry SM, Eriksson EA. Computed tomography pulmonary angiography: more than a screening tool for pulmonary embolus. J Crit Care. 2015; 30 (1): 196-200.

44. In $E$, Turgut $T$, Kalkan $M$, Karadağ $M O$, Dağlı MN. The relationship between computed tomography pulmonary angiography findings and right ventricular dysfunction in patients with pulmonary embolism. Turk Thorac J. 2014; 15: 117-121.

45. Scarsbrook A, Bradley K, Gleeson F, Groves AM, Yates SJ, Win T et al. Perfusion scintigraphy still has important role in evaluation of majority of pregnant patients with suspicion of pulmonary embolism. Radiology. 2007; 244 (2): 623-624.

46. Vedovati MC, Germini F, Agnelli G, Becattini C. Prognostic role of embolic burden assessed at computed tomography angiography in patients with acute pulmonary embolism: systematic review and meta-analysis. J Thromb Haemost. 2013; 11 (12): 2092-2102.

47. Schissler AJ, Rozenshtein A, Kulon ME, Pearson GD, Green RA, Stetson $\mathrm{PD}$ et al. CT pulmonary angiography: increasingly diagnosing less severe pulmonary emboli. PLoS One. 2013; 8 (6): e65669.

48. Cok G, Tasbakan MS, Ceylan M, Bayraktaroglu S, Duman S. Can we use $\mathrm{CT}$ pulmonary angiography as an alternative to echocardiography in determining right ventricular dysfunction and its severity in patients with acute pulmonary thromboembolism? Jpn J Radiol. 2013; 31 (3): 172-178.

49. Revel MP, Sánchez O, Couchon S, Planquette B, Hernigou A, Niarra $R$ et al. Diagnostic accuracy of magnetic resonance imaging for an acute pulmonary embolism: results of the "IRM-EP" study. J Thromb Haemost. 2012; 10 (5): 743-750.

50. Hosch W, Schlieter M, Ley S, Heye T, Kauczor HU, Libicher M. Detection of acute pulmonary embolism: feasibility of diagnostic accuracy of MRI using a stepwise protocol. Emerg Radiol. 2014; 21 (2): 151-158.

51. Jackowski C, Grabherr S, Schwendener N. Pulmonary thrombembolism as cause of death on unenhanced postmortem 3T MRI. Eur Radiol. 2013; 23 (5): 1266-1270

52. Hochhegger B, Ley-Zaporozhan J, Marchiori E, Irion K, Soares Souza Jr. A, Moreira J et al. Magnetic resonance imaging findings in acute pulmonary embolism. B J Radiol. 2011; 84: 282-287.

53. Sinzinger $H$, Rodrigues $M$, Kummer $F$. Ventilation/perfusion lung scintigraphy. Multiple applications besides pulmonary embolism. Hell J Nucl Med. 2013; 16 (1): 50-55.

54. Le Roux PY, Pelletier-Galarneau M, De Laroche R, Hofman MS, Zuckier LS, Roach P et al. Pulmonary scintigraphy for the diagnosis of acute pulmonary embolism: a survey of current practices in Australia, Canada, and France. J Nucl Med. 2015; 56: 1212-1217.

55. Perisinakis K, Seimenis I, Tzedakis A, Damilakis J. Perfusion scintigraphy versus 256-Slice CT angiography in pregnant patients suspected of pulmonary embolism: comparison of radiation risks. J Nucl Med. 2014; 55:1273-1280.

56. He J, Wang F, Dai HJ, Li M, Wang Q, Yao Z et al. Chinese multi-center study of lung scintigraphy and CT pulmonary angiography for the diagnosis of pulmonary embolism. Int J Cardiovasc Imaging. 2012; 28: 1799-1805.

57. Wang L, Kang W, Zu M, Zhang Q, Shen J, Wu L et al. Application of 128-slice spiral CT combination scanning in the diagnosis of embolisms in pulmonary arteries and lower extremity veins. Exp Ther Med. 2014; 7 (2): 401-404.

58. Brunot S, Corneloup O, Latrabe V, Montaudon M, Laurent F. Reproducibility of multi-detector spiral computed tomography in detection of sub-segmental acute pulmonary embolism. Eur Radiol. 2005; 15: 2057-2063.

59. Hiorns MP, Mayo JR. Spiral computed tomography for acute pulmonary embolism. Can Assoc Radiol J. 2002; 53 (5): 258-268.

60. Kamphuisen PW, Agnelli G. Spiral computed tomography is the firstline chest imaging test for acute pulmonary embolism: no. J Thromb Haemost. 2005; 3: 11-13.
61. Van Strijen MJ, De Monye W, Kieft GJ, Pattynama PM, Prins MH, Huisman MV. Accuracy of single-detector spiral CT in the diagnosis of pulmonary embolism: a prospective multicenter cohort study of consecutive patients with abnormal perfusion scintigraphy. J Thromb Haemost. 2005; 3 (1): 17-25.

62. Patel UB, Ward TJ, Kadoch MA, Cham MD. Radiographic features of pulmonary embolism: Hampton's hump. Postgrad Med J. 2014; 90 (1065): 420-421.

63. Abbas A, St Joseph EV, Mansour OMA, Peebles CR. Radiographic features of pulmonary embolism: Westermark and Palla signs. Postgrad Med J. 2014; 90: 422-423.

64. Stein PD, Matta F. Noninvasive imaging in pulmonary embolism according to age and gender. Clin Appl Thromb Hemost. 2014; 20 (2): 143-146.

65. Zhan ZQ, Wang CQ, Nikus KC, He CR, Wang J, Mao S et al. Electrocardiogram patterns during hemodynamic instability in patients with acute pulmonary embolism. Ann Noninvasive Electrocardiol. 2014; 19 (6): 543-551.

66. Icli A, Kayrak M, Akilli H, Aribas A, Coskun M, Ozer SF et al. Prognostic value of Tpeak-Tend interval in patients with acute pulmonary embolism. BMC Cardiovasc Disord. 2015; 15: 99.

67. Zhan ZQ, Wang CQ, Wang ZX, Nikus KC, Baranchuk A, Yuan RX et al. Significance of ST-segment deviation in patients with acute pulmonary embolism and negative T waves. Cardiol J. 2015; 22 (5): 583-589.

68. Aujesky D, Roy PM, Verschuren F, Righini M, Osterwalder J, Egloff M et al. Outpatient versus inpatient treatment for patients with acute pulmonary embolism: an international, open-label, randomised, non-inferiority trial. Lancet. 2011; 378 (9785): 41-48.

69. Spirk D, Willenberg T, Aujesky D, Husmann M, Hayoz D, Baldi T et al. Use of biomarkers or echocardiography in pulmonary embolism: the Swiss venous thromboembolism registry. Q J Med. 2012; 105 (12): 1163-1169.

70. Rodrigues AC, Guimaraes L, Guimaraes JF, Monaco C, Cordovil A, Lira E et al. Relationship of clot burden and echocardiographic severity of right ventricular dysfunction after acute pulmonary embolism. Int J Cardiovasc Imaging. 2015; 31: 509-515.

71. Rodrigues AC, Cordovil A, Mônaco CG, Guimarães LA, Oliveira WA, Fischer $\mathrm{CH}$ et al. Assessing prognosis of pulmonary embolism using tissue-Doppler echocardiography and brain natriuretic peptide. Einstein (Sao Paulo). 2013; 11 (3): 338-344.

72. Konstantinides SV, Torbicki A, Agnelli G, Danchin N, Fitzmaurice D, Galiè N et al. 2014 ESC Guidelines on the diagnosis and management of acute pulmonary embolism. Eur Heart J. 2014; 35: 3033-3069.

73. Gordon NT, Schreiber MA. Pulmonary emboli and deep vein thromboses: are they always part of the same disease spectrum? Mil Med. 2016; 181 (5 Suppl): 104-110.

74. Hogg K, Dawson D, Mackway-Jones K. Investigating pulmonary embolism in the emergency department with lower limb plethysmography: the Manchester Investigation of Pulmonary Embolism Diagnosis (MIOPED) study. Emerg Med J. 2006; 23: 94-98.

75. Sundaram PS, Padma S. Combined radionuclide phlebography and $\mathrm{V} / \mathrm{Q}$ scan in the assessment of iliac vein thrombosis and pulmonary embolism - A case report establishing a cause and effect relationship. Iran J Nucl Med. 2013; 21 (1): 36-39.

76. DeBakey ME, Schroeder GF, Ochsner A. Significance of phlebography in phlebothrombosis. JAMA. 2014; 311 (7): 741.

77. Lauque D, Maupas-Schwalm F, Bounes V, Juchet H, Bongard V, Roshdy A et al. Predictive value of the heart-type fatty acid-binding protein and the pulmonary embolism severity index in patients with acute pulmonary embolism in the emergency department. Acad Emerg Med. 2014; 21: 1143-1150.

78. Jo JY, Lee MY, Li JW, Rho BH, Choi WI. Leukocytes and systemic inflammatory response syndrome as prognostic factors in pulmonary embolism patients. BMC Pulm Med. 2013; 13: 74. 
79. Ozsu S, Abul Y, Yilmaz I, Ozsu A, Oztuna F, Bulbul Y et al. Prognostic significance of $\mathrm{PaO}_{2} / \mathrm{PaCO}_{2}$ ratio in normotensive patients with pulmonary embolism. Clin Respir J. 2012; 6 (2): 104-111.

80. Drent M, Cobben NA, Henderson RF, Wouters EF, van DieijenVisser M. Usefulness of lactate dehydrogenase and its isoenzymes as indicators of lung damage or inflammation. Eur Respir J. 1996; 9: 1736-1742.

81. Dursunoğlu N, Dursunoğlu D, Yıldız AI, Rota S. Evaluation of cardiac biomarkers and right ventricular dysfunction in patients with acute pulmonary embolism. Anatol J Cardiol. 2016; 16: 276282.

82. Gutte $\mathrm{H}$, Mortensen J, Jensen $\mathrm{CV}$, von der Recke P, Petersen $\mathrm{CL}$, Kristoffersen US et al. ANP, BNP and D-dimer predict right ventricular dysfunction in patients with acute pulmonary embolism. Clin Physiol Funct Imaging. 2010; 30: 466-472.
83. Bulj N, Potočnjak I, Sharma M, Pintarić H, Degoricija V. Timing of troponin T measurements in triage of pulmonary embolism patients. Croat Med J. 2013; 54: 561-568.

84. Khor YH, Smith R, McDonald CF. Suboptimal management of unfractionated heparin compared with low-molecular-weight heparin in the management of pulmonary embolism. Intern Med J. 2014; 44 (4): 339-344.

85. Hohner EM, Kruer RM, Gilmore VT, Streiff M, Gibbs H. Unfractionated heparin dosing for therapeutic anticoagulation in critically ill obese adults. J Crit Care. 2015; 30: 395-399.

86. Kostrubiec M, Łabyk A, Pedowska-Włoszek J, Dzikowska-Diduch O, Wojciechowski A, Garli n' ska M et al. Neutrophil gelatinaseassociated lipocalin, cystatin $\mathrm{C}$ and eGFR indicate acute kidney injury and predict prognosis of patients with acute pulmonary embolism. Heart. 2012; 98: 1221-1228. 